\title{
COMPOSICIÓN BOTÁNICA Y NUTRICIONAL DE LA DIETA DE Dinomys branickii (RODENTIA: DINOMYIDAE) EN LOS ANDES CENTRALES DE COLOMBIA
}

\section{BOTANICAL AND NUTRITIONAL COMPOSITION OF THE DIET OF Dinomys branickii (RODENTIA: DINOMYIDAE) IN CENTRAL ANDES OF COLOMBIA}

\begin{abstract}
José Luis González ${ }^{1}$, Karin Osbahr ${ }^{2}$
${ }^{1}$ Joven Investigador U.D.C.A Grupo de Investigación en Fauna Silvestre. Universidad de Ciencias Aplicadas y Ambientales U.D.C.A. Dirección para correspondencia: Calle 222 No. 55-37 Bogotá - D.C. E-mail: josgonzalez@udca.edu.co; ${ }^{2}$ Líder Grupo de Investigación en Fauna Silvestre. Universidad de Ciencias Aplicadas y Ambientales U.D.C.A. Dirección para correspondencia: Calle 222 No. 55-37 Bogotá - D.C. E-mail: kosbahr@udca.edu.co
\end{abstract}

Rev. U.D.C.A Act. \& Div. Cient. 16(1): 235 - 244, 2013

\section{RESUMEN}

El conocimiento de la nutrición de animales reúne aspectos ecológicos y de manejo de una especie. El objetivo principal de este trabajo fue determinar la composición botánica, el contenido nutricional expresado en base seca de la dieta y la preferencia de la Guagua loba o Pacarana, (Dinomys branickii) respecto al consumo de diferentes plantas en hábitat natural. En una localidad de la cordillera Central, se colectaron heces y material vegetal, identificado como recurso alimentario de la especie. Se obtuvieron fragmentos vegetales de las heces, mediante técnicas microhistológicas, lo que permitió identificar los diferentes tejidos epidérmicos. Los registros fotográficos permitieron comparar las cutículas del material vegetal con las de las heces. Se identificaron ocho familias, 10 géneros y 10 especies de plantas y las diferentes partes vegetativas (hoja, tallo, peciolo, rizoma e infrutescencia), en la dieta de la especie. El análisis del contenido nutricional de las plantas mostró valores altos en fibra, bajos en grasa con una importante concentración de cenizas. La Guagua loba es selectiva en el consumo de plantas y emplea una táctica de polifagia aprovechando, especialmente, plantas de los géneros Anthurium, Cyclanthus y Xanthosoma saggitifolium.

Palabras clave: Microhistología, recurso alimentario, pacarana, guagua loba, Andes, Colombia.

\section{SUMMARY}

The knowledge about wildlife nutrition meets ecological and management aspects of a particular species. The main ob- jective of this study was to determine the botanical composition and nutritional content expressed on a dry basis of the diet and the preference on different plant species consumed by the Pacarana (Dinomys branickii) in natural habitats. In Central Andes of Colombia, we collected feces and different food sources previously identified. We obtained plant fragments from feces using the microhistological technique, and identified different epidermal tissues. We compared photos of cuticles of plant material with the feces and identified eight families, 10 genera and 10 species of plants with different vegetative parts (leaf, stem, petiole, rhizome and infructescence). The analysis of plant nutritional contents showed high fiber and low fat with a high ash concentration. The species is selective against these resources and its strategy to select the diet is polyphagia including especially plants of the genus Anthurium, Cyclanthus and Xanthosoma saggitifolium.

Key words: Microhistology, food resource, Pacarana, Andes, Colombia.

\section{INTRODUCCIÓN}

La Guagua loba o Pacarana (Dinomys branickii) es el segundo roedor neotropical de mayor tamaño, cuya distribución geográfica abarca la cordillera de los Andes, desde Venezuela hasta Bolivia. En Colombia, se encuentra en los pisos térmicos medio y frío en las tres cordilleras, entre 1800 y $3400 \mathrm{msnm}$, donde habita zonas con pendientes pronunciadas y suelos rocosos (Saavedra-Rodríguez et al. 2012b).

La Guagua loba es una especie gregaria, esencialmente her- 
bívora, con un ciego desarrollado, que le permite digerir alimentos ricos en fibra y con hábitos selectivos, de acuerdo al contenido nutricional de la dieta (Osbahr \& Restrepo, 2001). Collins \& Eisenberg (1972), Woods (1984) y White \& Alberico (1992) sostienen que es herbívoro o fitófago estricto, que incluye en su dieta frutos de palmas, de hojas y de tallos; en Venezuela, Boher \& Marín (1988) registran el consumo de rizomas de plantas de la familia Araceae y Heliconiaceae; Osbahr (1998; 1999) reportó 15 géneros diferentes de plantas consumidas por $D$. branickii en su hábitat natural y Saavedra-Rodríguez et al. (2012b) realizaron el estudio de la dieta, a partir de la presencia de marcas de dientes en restos de plantas e identificaron 18 especies de plantas, consumidas por la especie.

La función ecológica de la Guagua loba puede consistir en la dispersión de semillas, como Ficus sp., de las que solamente consume la pulpa sin digerir las semillas. En muchos casos, las semillas pueden quedar enredadas en su pelo y caer eventualmente al suelo, para germinar lejos de sus árboles parentales (Osbahr, 1998). D. branickii se caracteriza por ser oportunista y consumidora de hojas, de tallos, de raíces y de frutos de diferentes plantas, modificando, de esta manera, el hábitat y, posiblemente, controlando el crecimiento excesivo de algunas plantas, como el chusque (Chusquea sp.), por lo que desempeña una función importante en la dinámica de sucesión de los bosques andinos (Osbahr, 1998; SaavedraRodríguez et al. 2012a).

El conocimiento de los hábitos alimentarios de animales silvestres permite obtener datos importantes de la composición botánica de la dieta y determinar su aporte nutricional. A la vez, la determinación bromatológica de las especies vegetales consumidas por una especie constituye una herramienta para definir las características de los hábitats idóneos para la sobrevivencia de las poblaciones (Mandujano, 1994; Vaughan, 2005). Además, la evaluación nutricional de los recursos que una especie consume en su hábitat natural provee una guía general para la formulación de dietas a poblaciones mantenidas en cautiverio, mejorando la calidad del recurso alimenticio ofrecido (Mautz et al. 1976).

El estudio de la dieta de animales, se hace con la técnica microhistológica, que se fundamenta en la identificación de estructuras, como células epidérmicas propiamente dichas, estomas, aparatos estomáticos, pelos, tricomas y glándulas (Catán et al. 2007). Estas estructuras se recuperan de heces, debido a que son pequeños fragmentos que no sufren cambios durante la digestión (Osbahr, 1999), permitiendo la identificación y la cuantificación de forrajes (Castellaro et al. 2007). Cuartas \& García (1996) aclaran que la mayor ventaja de esta técnica es que no es necesario el sacrificio de animales y no interfiere en su comportamiento.
El objetivo de este trabajo fue aportar información sobre la composición botánica de la dieta, las preferencias alimentarias hacia las diferentes partes vegetativas de las plantas y la composición nutricional del recurso alimentario consumido por $D$. branickii, en una zona boscosa de los Andes Centrales de Colombia. Los resultados contribuyen al desarrollo del plan de manejo elaborado para esta especie focal en el Sistema Regional de Áreas Protegidas del Eje Cafetero (SIRAPEC) (Saavedra-Rodríguez et al. 2012a).

\section{MATERIALES Y MÉTODOS}

Este estudio, se llevó a cabo entre agosto de 2009 y diciembre de 2010, en una localidad dentro del Distrito de Manejo Integrado de Suelos (DMS) Campoalegre, ubicada en la cuenca media del río San Eugenio (vereda La Paloma, sitios extremos Mina El Chaquiro: 449'02.1”N - 75³2’52.6”O y Garaje: $\left.4^{\circ} 50^{\prime} 50^{\prime \prime} \mathrm{N}-75^{\circ} 34^{\prime} 30^{\prime \prime} \mathrm{O}\right)$, a $7 \mathrm{~km}$ del municipio de Santa Rosa de Cabal, departamento de Risaralda, en el flanco occidental de la cordillera Central de Colombia, entre 1800 y 2600 msnm, en la zona amortiguadora del Parque Nacional Natural Los Nevados. El DMS tiene 20420ha, conformado por bosque continuo, parches de bosque secundario y matrices de plantaciones forestales, potreros y zona agrícola. La precipitación media anual es de $980 \mathrm{~mm}$ con comportamiento bimodal, con periodos menos lluviosos en diciembre - enero y julio - agosto (CARDER, 2009). Según el sistema de clasificación de Holdridge (1967), el área corresponde a la zona natural de vida bosque muy húmedo montano bajo (bmh-mb).

En la zona de estudio, se colectaron, siguiendo un muestreo preferencial (Knapp, 1984), las plantas referenciadas en trabajos anteriores por Osbahr $(1998 ; 1999)$ y confrontadas por Saavedra-Rodríguez et al. (2012b). La colecta se complementó con plantas que presentaron señales de forrajeo, por parte de $D$. branickii. Posterior a la identificación taxonómica, se incluyó el material de referencia con el número del colector en la colección biológica (Registro $053 \mathrm{IAvH}$ ), de la U.D.C.A. De cada una de las plantas, se colectó, adicionalmente, una muestra $(1 \mathrm{~kg})$ de material fresco, incluyendo todas y cada una de las partes vegetativas, para ser utilizadas en los análisis microhistológicos y bromatológicos.

Con la ayuda de un habitante de la zona, se identificaron indicios (comederos y madrigueras) de la presencia de Guagua loba y se realizó la búsqueda de letrinas, en un perímetro de $50 \mathrm{~m}$ alrededor de los indicios. Para cada letrina, se contó el total de cagarrutas encontradas y se conservaron en bolsas de cierre hermético (Ziploc $\left.{ }^{\circledR}\right)$, para transportarlas refrigeradas con bolsas de friogel (Calier $\left.{ }^{\circledR}\right)$ de $150 \mathrm{~g}$, desde el área de estudio hasta el laboratorio de la Unidad de Investigación en Fauna Silvestre de la U.D.C.A. 
Los fragmentos de hoja, de tallo, de pecíolo, de rizoma e infrutescencias de las especies vegetales colectadas y su recuperación en heces, se obtuvieron mediante la digestión química, utilizando la técnica descrita por Storr (1961) y modificada por Osbahr (1999). Se pesó el total de cada muestra y se extrajeron, previa homogenización en el caso de las heces, cinco gramos de cada una, para el análisis microhistológico y para la cuantificación de la dieta y de la oferta. Cada muestra, se montó en láminas portaobjetos y se identificaron al microscopio (40x) las células epidérmicas, estomas, aparatos estomáticos, pelos, tricomas y glándulas (Catán et al. 2007). Para cada estructura vegetal, se obtuvo un registro fotográfico, utilizando una cámara fotográfica digital. Finalmente, se compararon las microfotografías de los fragmentos vegetales recuperados en heces con las microfotografías de las láminas patrón obtenidas para cada especie vegetal colectada.

Para la cuantificación de los fragmentos vegetales, se siguió lo propuesto por Sparks \& Malechek (1968) y Holechek et al. (1982). Se montaron cinco láminas por cada muestra vegetal y de heces, observando 20 campos microscópicos por lámina, para un total de 100 campos visualizados por muestra. La observación sistemática, se realizó mediante un recorrido en líneas verticales paralelas de arriba hacia abajo, observando cada campo microscópico por separado.

La identificación de las especies vegetales presentes en las heces, se realizó registrando en todos los campos fragmentos vegetales conocidos y verificados con respecto a las láminas patrón de cada especie vegetal. Igualmente, se cuantificaron los fragmentos vegetales desconocidos, obteniendo la totalidad de los fragmentos presentes en las heces. Siguiendo a Cortés et al. (2003), se asumió que el número de fragmentos de plantas en las heces fue la misma que la ingerida y que todas las plantas fueron igualmente digeridas. Se utilizó la frecuencia de aparición (FA) equivalente al número de fragmentos (i) por especie vegetal, dividido por la sumatoria del total de fragmentos, multiplicado por 100 para realizar cálculos posteriores. Se asumió selectividad hacia determinada especie vegetal cuando FA es mayor en la dieta y evasión cuando FA es mayor en la oferta.

Para el análisis proximal de las especies vegetales consumidas por $D$. branickii, se secó cada una en un horno a $63^{\circ} \mathrm{C}$, registrando pesos diarios hasta obtener un peso constante (Hinnant \& Kothmann, 1998). La proteína total (PT), se determinó utilizando el método de digestión de Kjendahl; el extracto etéreo (EE), se obtuvo mediante destilación tipo Soxhelet con éter de petróleo (McDonald et al. 2006) y para la determinación de la fibra detergente neutra (FDN), se utilizó el método de Van Soest. La energía bruta (EB), se determinó en bomba calorimétrica. El contenido total de cenizas (C), se halló por incineración a $550^{\circ} \mathrm{C}$. El porcentaje de humedad, se determinó secando la muestra durante 48 horas a $63^{\circ} \mathrm{C}$ en un crisol hasta obtener un peso constante (Cuesta et al. 2000). Los datos obtenidos, se ajustaron en base seca (BS) (Herrera-Enciso et al. 1970).

Con el fin de evaluar preferencia o evasión de $D$. branickii sobre ítems alimentarios identificados, en el análisis microhistológico se ingresaron los datos al programa Havistat versión 1.0 (Montenegro \& Acosta, 2008), para calcular el Índice de Ivlev (1961) $\left(\mathrm{I}_{i}\right)$, cuyo rango varía entre -1 a 1 donde $\mathrm{I}_{i}>0=$ preferencia; $\mathrm{I}_{i}<0=$ evasión. El intervalo de confianza de Byers (Int. Byers ${ }_{i}$ ) cuyo rango va de -1 a $1<$ se aplicó, con el fin de confirmar los resultados del Índice de Ivlev y de establecer el uso de uno o más recursos (uso esperado < intervalo1 indica que prefiere; uso esperado > intervalo2 que evita e intervalo1 < uso esperado $<$ intervalo2 que evade) (Byers et al. 1984).

El porcentaje de cada especie vegetal en las muestras fecales, se determinó ajustando el porcentaje con el que una especie vegetal contribuyó al componente total de partes de planta (Schuette et al. 1998). Debido a que la digestibilidad de una materia prima o de un alimento representa la cantidad absorbida reportada en relación a la cantidad ingerida y puede ser estimada por diferencia en la fracción eliminada en las heces (De la Noüe et al. 1980), se realizó el cálculo del coeficiente de digestibilidad calculada aparente (CDA) del material, según el procedimiento reconocido para conejos (Villamide et al. 1998), con base en la siguiente ecuación:

$$
\mathrm{CDA}=[(\mathrm{CN}-\mathrm{EN}) \div \mathrm{CN}] \times 100
$$

Donde, $\mathrm{CN}=$ consumo del material y EN $=$ excreción del material.

Para establecer si existen diferencias significativas en los aportes de las plantas a la dieta evaluada, se realizó una prueba no paramétrica de Mann-Whitney U, para lo cual, se utilizó el paquete estadístico Statgraphics Plus 5.1.

\section{RESULTADOS Y DISCUSIÓN}

Durante el estudio, se colectaron en total 21 especies pertenecientes a 14 familias y 19 géneros diferentes de plantas de consumo potencial por la Guagua loba. Del listado inicial, ocho familias, diez géneros y diez especies pudieron ser verificados mediante la técnica microhistológica. A lo largo de los recorridos, se observaron señales de forrajeo en los tallos de las plantas Anthurim $\mathrm{sp}_{1}$, Bambusa sp. Xanthosoma saggittifolium, Hedychium heliotropa. c.f, y las hojas de Chamaedorea pinnatifrons. Igualmente, en repetidas ocasiones, se identificaron rizomas roídos de Gunnera pilosa. Durante el estudio, se encontraron tres especies de los géneros Bambusa, Hedychium y Xanthosoma no reportadas a la fecha, 
pero que se encuentran incluidas en la dieta de $D$. branickii. Las plantas de la familia Araceae constituyen uno de los recursos alimentarios más importantes para la Guagua loba en su hábitat natural, entre ellas tres especies del género Anthurium y una del género Xanthosoma (Tabla 1). En total, se identificaron 71 fragmentos vegetales diferentes, que presentaron variación de formas, de tamaño y de estructu- ras evidenciando las variaciones morfológicas de los tejidos epidérmicos entre las especies de plantas y entre las hojas, pecíolos, tallos, rizomas, e infrutescencias examinadas.

La recolección de heces en la localidad de estudio permitió la obtención de tres muestras (A, B y C; Tabla 2). SaavedraRodríguez et al. (2012a) reportan que la Guagua loba esta-

Tabla 1. Plantas colectadas como recurso alimentario potencial de la dieta de la Guagua loba (Dinomys branickii) en el DMS Campoalegre, Risaralda, Colombia. *Especies vegetales confirmadas en este y otros estudios (Saavedra-Rodríguez et al. 2012a, 2012b; Osbahr, 1999). El nombre común de las plantas es el dado por los pobladores de zonas andinas de Colombia.

\begin{tabular}{|c|c|c|c|c|c|c|c|}
\hline $\begin{array}{c}\mathrm{N}^{\circ} \mathrm{DE} \\
\text { COLECTOR }\end{array}$ & FAMILIA & GÉNERO & ESPECIE & $\begin{array}{l}\text { NOMBRE } \\
\text { COMÚN }\end{array}$ & $\begin{array}{c}\text { ESTE } \\
\text { ESTUDIO }\end{array}$ & $\begin{array}{l}\text { Saavedra } \\
\text { et al. }\end{array}$ & Osbahr \\
\hline JAM 016 & Apiaceae & $\begin{array}{l}\text { Myrrhiden- } \\
\text { dron }\end{array}$ & penneli & Arracachuela & & $*$ & * \\
\hline JAM 002 & Araceae & Anthurium & $\mathrm{sp}_{1}$ & $\begin{array}{l}\text { Bejuco de } \\
\text { árbol }\end{array}$ & * & $*$ & $*$ \\
\hline JAM 004 & Araceae & Anthurium & $\mathrm{sp}_{2}$ & Corazón & $*$ & $*$ & $*$ \\
\hline JAM 009 & Araceae & Anthurium & $\mathrm{sp}_{3}$ & $\begin{array}{l}\text { Anturio de } \\
\text { huevitos }\end{array}$ & $*$ & $*$ & $*$ \\
\hline JAM 005 & Araceae & Xanthosoma & saggitifolium & Rascadera & $*$ & & \\
\hline JAM 010 & Arecaceae & Genoma & sp. & Palma & & $*$ & \\
\hline JAM 012 & Arecaceae & $\begin{array}{l}\text { Chamaedo- } \\
\text { rea }\end{array}$ & pinnatifrons & Bodenillo & $*$ & & \\
\hline JAM 003 & Cyclanthaceae & Cyclanthus & sp. & Cestillo & $*$ & $*$ & $*$ \\
\hline JAM 020 & $\begin{array}{l}\text { Dryopterida- } \\
\text { seae }\end{array}$ & Dryopteris & sp. & Helecho & & $*$ & $*$ \\
\hline JAM 015 & Graminaceae & Axonopus & scoparius & $\begin{array}{l}\text { Pasto Impe- } \\
\text { rial }\end{array}$ & & & \\
\hline JAM 013 & Gunneraceae & Gunnera & pilosa. & $\begin{array}{l}\text { Mata de } \\
\text { Pantano }\end{array}$ & $*$ & $*$ & \\
\hline JAM 018 & Heliconiaceae & Heliconia & burleana. & Platanillo & & $*$ & $*$ \\
\hline JAM 022 & Marattiaceae & Marattia & sp. & Helecho & & & \\
\hline JAM 007 & Piperaceae & Piper & potomorphe & Cordoncillo & & & \\
\hline JAM 014 & Poaceae & Bambusa & sp. & Bejuco Biche & $*$ & & $*$ \\
\hline JAM 017 & Poaceae & $\begin{array}{l}\text { Indetermi- } \\
\text { nada }\end{array}$ & Indeterminada & & & & \\
\hline JAM 019 & Poaceae & Gynerium & sp. & Cañabrava & & & \\
\hline JAM 021 & Poaceae & Panicum & Indeterminada & Chusque & & & \\
\hline JAM 001 & Polypodiaceae & Pteridium & sp. & Helecho & & $*$ & \\
\hline JAM 008 & Solanaceae & $\begin{array}{l}\text { Indetermi- } \\
\text { nada }\end{array}$ & Indeterminada & & $*$ & $*$ & \\
\hline JAM 006 & Zingiberaceae & Hedychium & heliotropa c.f & Fruto rojo & $*$ & & \\
\hline
\end{tabular}


blece letrinas ubicadas entre 25 y $60 \mathrm{~m}$ de distancia de las madrigueras y las zonas de forrajeo se establecen de 10 a $150 \mathrm{~m}$, las cuales, se ubican en terrenos rocosos, con pendiente abrupta, en parches de bosque de vegetación abundante y cercanos a zonas ribereñas, lo que permite afirmar que la zona donde se realizó la recolección de heces es utilizada por $D$. branickii para forrajear y para resguardo en refugios. Osbahr (2010) realizó una estimación de la tasa de defecación en la especie y registró un promedio de cagarru- tas/individuo/día en hembras, de 14,6 y el promedio de cagarrutas/individuo/día en machos, de 11,8. Comparando lo mencionado anteriormente con el numero de cagarrutas por muestra que se encontraron en la localidad (Tabla 2), se deduce que las muestras A y B proceden de un solo individuo, ya que la especie no necesariamente forma grupos familiares si no también se les puede ver solitarios o en parejas (Woods, 1984). Los grupos familiares que conforma $D$. branickii están compuestos por dos a cinco individuos (Boher \& Marín,

Tabla 2. Muestras de heces de Guagua loba (Dinomys branickii) colectadas en el DMS Campoalegre, Santa Rosa de Cabal, Risaralda, Andes Centrales de Colombia.

\begin{tabular}{|c|c|c|c|}
\hline MUESTRA & No DE CAGARRUTAS & PESO DE LA MUESTRA (g) & LUGAR DE COLECTA \\
\hline A & 10 & 46 & $\begin{array}{c}\text { Media montaña, alta pendiente cercana } \\
\text { a plantaciones forestales }\end{array}$ \\
\hline B & 14 & 98 & $\begin{array}{c}\text { Alta pendiente, zona rocosa cercana a } \\
\text { fuentes de agua }\end{array}$ \\
\hline C & 25 & 230 & $\begin{array}{c}\text { Media montaña, vegetación de bosque } \\
\text { densa, baja pendiente cercana a tron- } \\
\text { cos caídos }\end{array}$ \\
\hline
\end{tabular}

1988; Saavedra-Rodríguez et al. 2012b) y el promedio de cagarrutas/letrina/día de un grupo de cinco individuos es de 59,7 (Osbahr, 2010); por lo tanto, se deduce que la muestra $\mathrm{C}$ es procedente de un grupo de más de tres individuos.

En las heces, se identificaron y se cuantificaron en total 415 $(48,15 \%)$ fragmentos vegetales conocidos y 447 (51,85\%) desconocidos. Los fragmentos no identificados podrían corresponden a 11 especies vegetales colectadas, pero no verificadas en este estudio (Tabla 1), que podrían presentar una posible mayor digestibilidad; esto explicaría la sub o sobreestimación en la cuantificación de fragmentos vegetales (Catán et al. 2007). De hecho, D. branickii forrajea tallos y hojas jóvenes, por lo que el estado fenológico de las plantas, en el momento de la ingesta, afecta igualmente la digestibilidad y la presencia de fragmentos vegetales identificables en las heces; por lo tanto, al no contar con un factor de corrección especie-específico que compense, se asume que la parcialidad en los análisis microhistológicos de las heces asociada a la digestión diferencial de los forrajes es igual (Leslie et al. 1983).

De acuerdo a los resultados obtenidos, $D$. branickii selecciona o evade el recurso alimentario disponible, lo cual, se manifiesta al comparar la frecuencia de aparición (FA) del total de fragmentos vegetales en la oferta de la muestra recuperada en heces. Para Anthurium sp., Cyclanthus sp., Xanthosoma saggitifolium, Gunnera pilosa y Bambusa sp. FA es mayor en la dieta que en la oferta (Figura 1a), por lo que se considera que la especie es selectiva frente a estos recursos, a la vez, que desde la escala ecológica, su estrategia para seleccionar la dieta obedece a una táctica de polifagia (Sherry, 1990). De hecho, los fragmentos vegetales recuperados en heces registraron diferentes estructuras vegetativas consumidas por $D$. branickii, donde el $42,8 \%$ corresponde a las hojas y el $35,7 \%$ a los tallos, mientras que los rizomas, infrutescencias y peciolos, ocupan el $7,1 \%$.

Se encontraron diferencias estadísticamente significativas entre el consumo de tallos y rizoma ( $p \leq 0,05$ test de $U-$ Mann - Withney), es decir, $D$. branickii utiliza de manera diferencial las plantas evaluadas, consumiendo únicamente el tallo de Anthurium $\mathrm{sp}_{1}$, H. heliotropa c.f. y Bambusa sp., las hojas de la especie de la familia Solanaceae, Anthurium $\mathrm{sp}_{3}$. y C. pinnatifrons, y el rizoma de G. pilosa. En cambio Cyclanthus sp., Anthurium $\mathrm{sp}_{2}$ y $X$. saggitifolium, se caracterizaron por el consumo mixto de más de una estructura de la planta (Figura 1b); sin embargo, el cálculo del índice Ivlev (Ii) junto con el intervalo de confianza de Byers (Int Byers $i$ ) indica una clara preferencia hacia el pecíolo de Anthurium $\mathrm{sp}_{2}$., el tallo de Bambusa sp y Cyclanthus sp., el rizoma de $G$. pilosa y la hoja e infrutescencia de $X$. saggitifolium (Tabla 3).

Debido a que el estudio se limitó al análisis de las plantas que pudieron ser verificadas en las heces, el conocimiento parcial de la dieta permite afirmar que la Guagua loba consume alimentos altos en fibra, bajos en grasa con una 

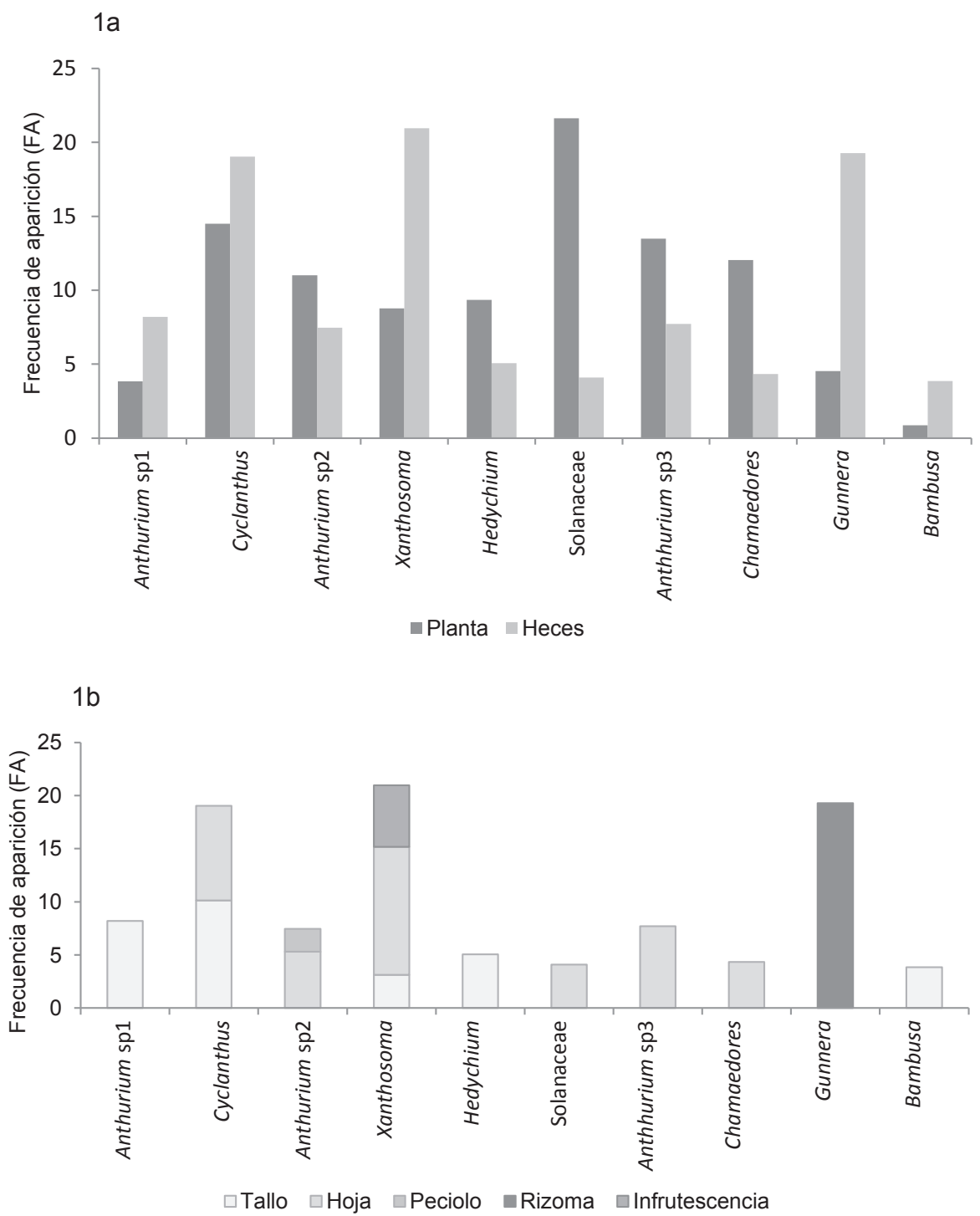

Figura 1. Preferencias alimentarias de D. branickii con base en los fragmentos recuperados en heces. (1a) Selectividad observada en relación a la oferta del recurso. 1(b) Uso diferencial del recurso alimentario. $T=$ Tallo, $H=$ Hoja, $P=P e c i o l o, ~ R$ $=$ Rizoma, IN = Infrutescencia.

importante concentración de cenizas (Tabla 3). El porcentaje promedio de nutrientes en base seca presentes en los diferentes alimentos consumidos con un coeficiente de digestibilidad aparente (CDA) de 88,48 $\pm 12,86$ equivale a proteína total (PT) 11,36 \pm 4,8; grasa (EE) 3,16 \pm 2,0; fibra detergente neutra (FDN) 60,7 \pm 14 ; cenizas (C) 15,3 \pm 6,0 y energía bruta (EB) 3,876 kcal/g $\pm 0,29$. En general, los tallos contienen niveles superiores al $38,8 \%$ de FDN, donde sobresalen Bambusa sp. y $H$. heliotropa c.f., con valores porcentuales mayores al $80 \%$. El consumo de componentes fibrosos es una fuente de energía, debido a que $D$. branickii es un herbívoro de fermentación cecal (Osbahr \& Restrepo, 2001), por lo que está en capacidad de degradar la fibra de los alimentos, produciendo ácidos grasos volátiles. Los tallos también le permiten a la especie roer, sujetar y tomar el alimento acorde con sus características físicas, morfológicas y comportamentales (Osbahr, 1998). Mejía et al. (2009) reportan que el Género Bambusa contiene lignanos, componentes de la fibra que exhiben un número de características importantes que están en investigación, pero al parecer tienen actividad antibacteriana, antifúngica y antiviral. Existe la posibilidad de que $D$. branickii obtenga estas sustancias, a partir de los tallos que consume de esta especie vegetal. A su vez, los tallos de Anthurium $\mathrm{sp}_{1}$ y de $X$. saggitifolium contienen los máximos niveles de cenizas (> $25 \%$ ), esenciales para la formación de huesos, de enzimas, 
Tabla 3. Contenido nutricional en base seca del recurso alimentario aprovechado por $D$. branickii. $I i=$ Índice de preferencia Ivlev; Int Byers $i=$ Intervalo de confianza; CDA = Coeficiente de digestibilidad aparente; $M S=$ Materia seca; PT = Proteína total; EE = Extracto etéreo; $\mathrm{C}=$ Cenizas; FDN = Fibra digestible neutra; EB = Energía bruta; SD = Sin determinar.

*** Prefiere, ** Evita, * Indiferente

\begin{tabular}{|c|c|c|c|c|c|c|c|c|c|c|c|}
\hline $\begin{array}{c}\text { NOMBRE } \\
\text { CIENTÍFICO }\end{array}$ & $\begin{array}{c}\text { ES- } \\
\text { TRUCTURA } \\
\text { COMSU- } \\
\text { MIDA }\end{array}$ & Ii & \multicolumn{2}{|c|}{ Int Byers i } & \multirow{2}{*}{$\begin{array}{l}\text { CDA } \\
91,15\end{array}$} & \multirow{2}{*}{$\begin{array}{r}\text { \%MS } \\
6,36\end{array}$} & \multirow{2}{*}{$\begin{array}{c}\% \mathrm{PT} \\
12\end{array}$} & \multirow{2}{*}{$\begin{array}{r}\% \mathrm{EE} \\
2,9\end{array}$} & \multirow{2}{*}{$\begin{array}{c}\% \mathrm{C} \\
25\end{array}$} & \multirow{2}{*}{$\begin{array}{c}\text { \%FDN } \\
55,9\end{array}$} & \multirow{2}{*}{$\begin{array}{c}\begin{array}{c}\mathrm{EB} \\
\mathbf{k c a l} / \mathbf{g}\end{array} \\
3,735\end{array}$} \\
\hline Anthurium $\mathrm{sp}_{1}$. & Tallo & 0,8 & $0,04 * *$ & $\begin{array}{c}0,15- \\
0,38\end{array}$ & & & & & & & \\
\hline \multirow{2}{*}{ Anthurium $\mathrm{sp}_{2}$. } & Hoja & 0,1 & $0,11^{*}$ & $\begin{array}{c}0,05- \\
0,20\end{array}$ & 97,07 & 13,26 & 16,7 & 3,7 & 15,9 & 57,4 & 3,955 \\
\hline & Peciolo & 0,8 & $0,11 * * *$ & $\begin{array}{c}0,94- \\
1,06\end{array}$ & 99,59 & 10,41 & 6,8 & 1,2 & 17,1 & 65,8 & 3,835 \\
\hline Anthurium $\mathrm{sp}_{3}$. & Hoja & 0,1 & $0,13 *$ & $\begin{array}{c}0,10- \\
0,27\end{array}$ & 88,57 & 14,37 & 17,4 & 4,4 & 18,2 & 53,4 & 3,651 \\
\hline Bambusa sp. & Tallo & 0,9 & $0,01 * * *$ & $\begin{array}{c}0,04- \\
0,21\end{array}$ & 95,24 & 13,42 & 6,2 & 0,9 & 9,3 & 82,9 & 4,165 \\
\hline $\begin{array}{c}\text { Chamaedorea } \\
\text { pinnatifrons }\end{array}$ & Hoja & $-0,1 * *$ & $0,12 *$ & $\begin{array}{c}0,00- \\
0,00\end{array}$ & 95,87 & 13,79 & 14,5 & 3,6 & 10,6 & 65,4 & SD \\
\hline \multirow{2}{*}{ Cyclanthus sp. } & Hoja & 0,2 & $0,15 *$ & $\begin{array}{c}0,12- \\
0,30\end{array}$ & 97,02 & 17,95 & 14,3 & 2,6 & 10,1 & 70,8 & 4,216 \\
\hline & Tallo & 0,4 & $0,15 * * *$ & $\begin{array}{l}0,21- \\
0,46\end{array}$ & 80,37 & 14,19 & 7,5 & 1,1 & 12,7 & 64,3 & 3,825 \\
\hline Gunnera pilosa & Rizoma & 0,9 & $0,05 * * *$ & $\begin{array}{c}0,99- \\
1,01\end{array}$ & 50,92 & 16,93 & 5 & 1,4 & 7,1 & 41,8 & 3,703 \\
\hline $\begin{array}{l}\text { Hedychium } \\
\text { heliotropa c.f. }\end{array}$ & Tallo & 0,3 & $0,09 *$ & $\begin{array}{c}0,07- \\
0,26\end{array}$ & 78,13 & 13,12 & 5,6 & 1,9 & 9,2 & 86 & 3,973 \\
\hline Solanaceae & Hoja & $-0,4 * *$ & $0,22 * *$ & $\begin{array}{l}0,03- \\
0,16\end{array}$ & 80,23 & 11,66 & 17,4 & 4 & 15,5 & 69,5 & 3,964 \\
\hline \multirow{3}{*}{$\begin{array}{l}\text { Xanthosoma } \\
\text { saggitifolium }\end{array}$} & Hoja & 0,5 & $0,09 * * *$ & $\begin{array}{c}0,19- \\
0,38\end{array}$ & 94,35 & 13,94 & 17 & 7,9 & 15,5 & 47,4 & 4,400 \\
\hline & $\begin{array}{c}\text { Infrutescen- } \\
\text { cia }\end{array}$ & 0,8 & $0,09 * * *$ & $\begin{array}{c}0,98- \\
1,02\end{array}$ & 94,71 & 9,44 & 12 & 6,4 & 21,7 & 51,8 & 3,856 \\
\hline & Tallo & 0,1 & $0,09 *$ & $\begin{array}{c}0,02- \\
0,18\end{array}$ & 95,61 & 4,91 & 6,7 & 2,4 & 26,5 & 38,8 & 3,233 \\
\hline
\end{tabular}

de hormonas y de electrolitos, que ayudan al mantenimiento homeostático de los organismos (McDonald, 2006). El pecíolo, se caracteriza por contenidos altos en cenizas y de fibra, mientras que las hojas son las estructuras que contienen mayor porcentaje de proteína $(>16,7 \%)$ y grasa (> 3,7\%). La infrutescencia es una estructura que presenta niveles significativos de proteína y de ceniza. El rizoma de $G$. pilosa aporta niveles importantes de energía bruta (Tabla 3); por lo general, estas estructuras son ricas en almidón, lo que ayuda a obtener energía de rápida absorción y liberación, a la vez que pueden presentar bajos contenidos de proteína, pero gran variedad de vitaminas y minerales.

Las fuentes proteicas de origen vegetal que presenten contenidos mayores al $15 \%$ de proteína indican una buena contribución a la dieta; sin embargo, el intervalo de confianza de Byers (Int Byers $i$ ) indica niveles bajos de uso de las hojas con elevados contenidos en proteína (Tabla 3), siendo 
la excepción las hojas de Xanthosoma. Sarria et al. (1999) reportan que estas hojas aportan niveles significativos de proteína y de minerales, un adecuado balance de aminoácidos, con respecto a la lisina y un importante contenido de potasio, lo cual, explicaría la preferencia de $D$. branickii por este recurso, aunque las hojas de esta planta, a su vez, son deficientes en fósforo y en sodio y registran la presencia de factores antinutricionales, como oxalatos de calcio. El aporte fundamental de la proteína en la dieta para cumplir con los requerimientos de una especie es el contenido de aminoácidos, puesto que estos son esenciales para el funcionamiento del organismo. De tal manera que los valores obtenidos mediante el análisis bromatológico no permiten establecer con certeza si la especie prefiere determinado recurso con respecto a este nutriente en general o a la composición específica de los aminoácidos esenciales (Sedinger, 1984). En el caso de $D$. branickii, aun cuando ingiera una dieta de baja calidad nutricional, optimiza las fuentes de aminoácidos para cubrir sus necesidades, puesto que la fermentación cecal le permite aprovechar la proteína bacteriana, como una fuente importante de aminoácidos (Barboza et al. 2009).

Si bien la Teoría del Forrajeo Óptimo (McArthur \& Pianka, 1966) enfatiza la ganancia en la tasa energética como elemento importante en la selección de la dieta, con el fin de generar un balance positivo de energía, este concepto no explica por sí solo las diferencias encontradas en los índices de preferencia por planta (Tabla 3). Estudios realizados en cautiverio indican, igualmente, que la especie es de hábitos selectivos, donde la proporción proteína/grasa en la dieta juega un papel importante (Osbahr \& Restrepo, 2001), a la vez, que de acuerdo con López et al. (2000) es muy notable la proporción de tiempo que la especie invierte en la alimentación (31\%), la cual, aunque es baja en frecuencia promedio (2,92/hora), es de duración promedio considerable (6,43 minutos). D. branickii, además de explorar los parches de alimentación, arregla la comida previa ingestión y la roe lentamente en vez de engullirla. Estas características propias de la historia natural de la especie sin duda alguna influyen sobre la selección de la dieta.

Los resultados obtenidos permiten inferir que los micronutrientes, los efectos asociativos de una dieta mixta y los mecanismos de asimilación y metabolización de nutrientes (Bozinovic \& Martínez del Río, 1996) juegan un papel importante en las decisiones de forrajeo de la Guagua loba seleccionando su alimento, de acuerdo a la composición nutricional que le puedan ofrecer los recursos disponibles para sus procesos fisiológicos. En el caso de los herbívoros, la proteína y los carbohidratos se encuentran entre los nutrientes más importantes, puesto que sus concentraciones en las plantas son altamente variables y, en ocasiones, limitantes, dependiendo del tipo de planta, la edad y de sus condiciones de crecimiento (Bernays \& Chapman, 1994). En muchos casos, la mezcla apropiada de estos nutrientes se obtiene a través de la regulación de la cantidad ingerida de un alimento en particular o ingiriendo una mezcla de alimentos (Raubenheimer \& Simpson, 2004); por lo tanto, un herbívoro oportunista, como es el caso de $D$. branickii, tiene la capacidad de alcanzar su meta de consumo alternando entre más de un alimento que, por sí solo, es nutricionalmente subóptimo, pero que en conjunto son complementarios, es decir, que al consumir diferentes especies vegetales y estructuras de las plantas obtiene una dieta equilibrada que le proporciona una capacidad amortiguadora frente a la calidad diferencial del recurso disponible en el hábitat (Behmer \& Joern, 2008). La palatibilidad, definida como las características de la planta que estimulan la respuesta alimenticia selectiva y que está relacionada con los componentes químicos, tales como los contenidos de proteína, los niveles de ceniza, el contenido de humedad y la digestibilidad, puede ser considerada como otro elemento que induzca a $D$. branickii a seleccionar, con mayor preferencia, algunos componentes de la dieta (Robbins, 2001).

La aproximación obtenida en este estudio establece una selección y una evasión de algunos componentes de la dieta; no obstante, para determinar si la especie hace un uso óptimo de los recursos alimentarios evaluados, es necesario considerar en futuros estudios su distribución espacial, para así obtener una mejor aproximación al gasto energético requerido durante el desplazamiento, para encontrar los alimentos que ofrezcan los nutrientes adecuados para el desarrollo, el sostenimiento y la reproducción de las poblaciones de $D$. branickii.

Agradecimientos: A Hernando Estupiñán, por su colaboración en la identificación de las especies vegetales; a Aurora Cuesta, Margoth Arévalo y al personal de los Laboratorios de Nutrición Animal y Fitotecnia de la U.D.C.A, a los habitantes de la vereda La Paloma, por su disposición y su colaboración permanente, a Carlos A. Saavedra-Rodríguez, por su apoyo investigativo y asistencia en campo; a la Zoological Society for the Protection of Species and Populations (ZGAP), por brindarnos el apoyo económico. Conflicto de intereses: El manuscrito fue preparado y revisado con la participación de todos los autores, quienes declaramos que no existe ningún conflicto de intereses que ponga en riesgo la validez de los resultados presentados.

\section{BIBLIOGRAFÍA}

1. BARBOZA, P.S.; PARKER, K.L.; HUME, I.D. 2009. Integrative Wildlife Nutrition. Springer Verlag Berlin Heidelberg 342p. 
2. BEHMER, S.T.; JOERN, A. 2008. Coexisting generalist herbivores occupy unique nutritional feeding niches. Proc. Nal Acad. Sci. 105(6):1977-1982.

3. BERNAYS, E.A.; CHAPMAN, R.F. 1994. Host Plant Selection by Phytophagous Insects. Chapman \& Hall, New York. p.7-9.

4. BOHER, S.; MARÍN, B. 1988. El pacarana (Dinomys branickii) en Venezuela. Natura. 84:14-18.

5. BOZINOVIC, R.H.; MARTÍNEZ DEL RÍO, C. 1996. Animals eat what they should not: why do they reject our foraging models? Rev. Chil. Historia Natural. 69:1520.

6. BYERS, C.R.; STEINHORST, C.K.; KRAUSMAN, P.R. 1984. Clarification of a technique for analysis of utilization-availability data. J. Wildlife Manag. 48:10501053.

7. CASTELLARO, G.; SQUELLA, F.; ULLRICH, T.; LEÓN, F.; RAGGI, A. 2007. Algunas técnicas microhistológicas utilizadas en la determinación de la composición botánica de dietas de herbívoros. Agr. Técn. 67(1):86-93.

8. CATÁN, A.; DEGANOA, C.A.; WERENITZKYA, D. 2007. Evaluación de criterios de lectura microhistológica para la cuantificación de Sphaeralcea bonariensis (Cav.), Pl Lorentz en mezclas manuales. Técn. Pec. México. 45(1):77-83.

9. COLLINS, L.R.; EISENBERG, J.F. 1972. Notes on the behavior and breeding of pacaranas in captivity. Int. Zoo Yearbook. 12:108-114.

10. CORPORACIÓN AUTÓNOMA REGIONAL DE RISARALDA (CARDER). 2009. Sistema Departamental de Áreas Protegidas de Risaralda. 30p.

11. CORTÉS, A.; MIRANDA, E.; RAU, J.R.; JIMÉNEZ, J.E. 2003. Feeding habits of guanacos Lama guanicoe in the high Andes of north-central Chile. Acta Theriologica 48:229-237.

12. CUARTAS, P.; GARCÍA, R. 1996. Review of available techniques for determining the diet of large herbivores from their feces. Oecologia Montana 5:47-50.

13. CUESTA, A.P.; JIMÉNEZ, D.A.; MATEUS, G. 2000. Características nutricionales y antinutricionales de especies no leguminosas como alimentación natural de "rebusque" de oveja Africana. Rev. U.D.C.A Act. \& Div. Cient. 3(1):13-20.

14. DE LANOÜE, J.; CHOUBERT, G.; PAGNIEZ, B.; BLANC, J.M.; LUQUET, P. 1980. Digestibilité chez la truite arc-en-ciel (Salmo gairdneri) lors de l'adaptation à un nouveau regime alimentaire. Can. J. Fish. Aquat. Sci. 37:2218-2224.

15. IVLEV, V.S. 1961. Experimental ecology of the feeding of fishes. Yale University Press, New Haven. 302p.

16. HERRERA-ENCISO, H.; GALLO-CARDONA, J.T.; MANER, J.H.; CEBALLOS-BUENO, E. 1970. Análisis químico-bromatológico de algunas materias primas colombianas empleadas en nutrición animal. Instituto Colombiano Agropecuario. 29p.

17. HINNANT, R.T.; KOTHMANN. M.M. 1998. Collecting, drying and preserving feces for chemical and microhistological analysis. J. Range Manag. 41(2):168171.

18. HOLECHEK, J.L.; VAVRA, M.; PIEPER, R.D. 1982. Botanical composition determination of range herbivore diets: A review. J. Range Manag. 35(3):309-315.

19. HOLDRIDGE, L.R. 1967. Life zone ecology. Trop. Sci. Center, San José, Costa Rica. 206p.

20. KNAPP, R. 1984. Sampling methods and taxon analysis in vegetation science. Ed. Springer. 388p.

21. LESLIE, D.M.; VAVRA, M.; STARKEY, E.E.; SLATER, C. 1983. Correcting for differential digestibility in microhistological analyses involving common coastal forages of the Pacific Northwest. J. Range Manag. 36:730-732.

22. LÓPEZ, L.; LÓPEZ, I.; MORA, J.; OSBAHR, K. 2000. Estudio Preliminar del comportamiento de Dinomys branickii (Peters, 1873) en cautiverio. Rev. U.D.C.A Act. \& Div. Cient. 3(1):28- 35.

23. McDONALD, P.; EDWARDS, R.A.; GREENHALGH, J.F.D.; MORGAN, C.A. 2006. Nutrición Animal. $6^{\text {a }}$ ed. 600p.

24. MANDUJANO, S. 1994. Método para evaluar el hábitat del Venado Cola Blanca en un bosque de coníferas. En: Vaughan, C.; Rodríguez, M. (eds.) Ecología y manejo del Venado Cola Blanca en México y Costa Rica. Ed. Universidad Nacional Autónoma de México. p.283-297. 
25. MAUTZ, W.W.; SILVER, H.; HOLTER, J.B.; HAYES, H.H.; URBAN, W. 1976. Digestibility and related nutritional data for seven northern deer browse species. J. Wildlife Manag. 40:630-683.

26. McARTHUR, R.H.; PIANKA, E.R. 1966. On optimal use of a patchy environment. Am. Nat. 100:603-609.

27. MEJÍA, A.I.; GALLARDO, C.; VALLEJO, J.J.; RAMÍREZ, G.; ARBOLEDA, C.; DURANGO, E.S.; JARAMILLO, F.A.; CADAVID, E. 2009. Plantas del Género Bambusa: importancia y aplicaciones en la industria farmacéutica, cosmética y alimentaria. Vitae. 16(3):396405.

28. MONTENEGRO, J.; ACOSTA, A. 2008. Programa innovador para evaluar uso y preferencia de hábitat. Univ. Scient. 13(2):208-217.

29. OSBAHR, K. 1998. Guía para la conservación de la "guagua loba o pacarana" (Dinomys branickii). Bernal, H.Y.; Farfán, M. (eds.) SECAB, Ciencia y tecnología No. $651^{\text {a }}$ edición. Convenio Andrés Bello. 43p.

30. OSBAHR, K. 1999. Identificación de plantas consumidas por Agouti taczanowskii y Dinomys branickii a partir de fragmentos vegetales recuperados en heces. Rev. U.D.C.A Act. \& Div. Cient. 2(2):42-49.

31. OSBAHR, K. 2010. Evaluación de la tasa de defecación y del uso de letrinas en la guagua loba (Dinomys branickii: Rodentia - Dinomyidae). Rev. U.D.C.A Act. \& Div. Cient. 13(1):35-45.

32. OSBAHR, K.; RESTREPO, D. 2001. Determinación de calcio, hierro, proteína y otros requerimientos de nutrientes de Dinomys branickii. Rev. U.D.C.A Act. \& Div. Cient. 4(1):44-55.

33. RAUBENHEIMER, D.; SIMPSON, S.J. 2004. Organismal stoichiometry: quantifying nonindependence among food components. Ecol. 85:1203-1216.

34. ROBBINS, C.T. 2001. Wildlife feeding and Nutrition. Academic Press INC 352p.

35. SAAVEDRA-RODRÍGUEZ, C.A.; OSBAHR, K.; ROJAS, V. 2012a. Plan de conservación y manejo de la Guagua loba (Dinomys branickii). Sistema Regional de Áreas Protegidas Eje Cafetero. CARDER-WCS-Colombia, 60p.
36. SAAVEDRA-RODRÍGUEZ, C.A.; KATTAN, G.; OSBAHR, K.; HOYOS, J.G. 2012b. Multiscale patterns of hábitat and space use by the Pacarana Dinomys branickii: Factors limiting its distribution and abundance. Endang. Spec. Res. 16:273-281.

37. SARRIA, P.; PASCAL, L.; LONDOÑO, A.; BOTERO, M. 1999. Valor nutricional de algunas forrajeras para la alimentación de monogástricos. Universidad Nacional de Colombia Sede Palmira. 128p.

38. SCHUETTE, J.R.; LESLIE Jr., D.M.; LOCHMILLER, R.L.; JENKS, J.A. 1998. Diets of hartebeest and roan antelope in Burkina Faso: Support of the long-faced hypothesis. J. Mammal. 79(2):426-436.

39. SEDINGER, J.S. 1984. Protein and amino acid composition of tundra vegetation in relation to nutritional requirements. J. Wildlife Manag. 48(4):1128-1136.

40. SHERRY, T.W. 1990. When are birds dietarily specialized? Distinguishing ecological from evolutionary approaches. Studies Avian Biol. 13:337-352.

41. SPARKS, D.R.; MALECHEK, J.C .1968. Estimating percentage dry weight in diets using microscopic technique. J. Wildlife Manag. 21:264-265.

42. STORR, G.M. 1961. Microscopic analysis of faeces, a technique for ascertaining the diet of herbivorous mammals. J. Biol. Sci. 14:157-164.

43. VILLAMIDE, M.J.; MAERTENS, L.; DE BLAS, C.; PEREZ, J.M. 1998. Feed evaluation. En: DE BLAS \& WISEMAN (eds.). The nutrition of the rabbit. CAB International Publication Co. Wallinford. p.89-101.

44. VAUGHAN, C. 2005. Ventajas y desventajas de las reintroducciones. Mesoam. 10(2):61-64.

45. WHITE, T.G.; ALBERICO, M.S. 1992. Dinomys branikii. Mammalian Species No. 410 : 1- 5. The American Society of Mammalogists, USA.

46. WOODS, C.A. 1984. Hystricognath rodents.En: Anderson, S.; Jones, J.K. (eds.) Orders and families of recent mammals of the world. Ed. John Wiley and Sons. (New York). p.389-446.

Recibido: Noviembre 21 de 2012

Aceptado: Abril 4 de 2013 